\title{
On the measure of axial symmetry with respect to folding for parallelograms
}

\author{
Monika Nowicka
}

Received: 22 June 2010 / Accepted: 18 April 2011 / Published online: 4 May 2011

(C) The Author(s) 2011. This article is published with open access at Springerlink.com

\begin{abstract}
Let $C_{m}$ be a subset of a planar convex body $C$ cut off by a straight line $m$, which remains in $C$ after folding it along $m$. The maximum $\operatorname{masf}(C)$ of the ratio of the double area of $C_{m}$ to the area of $C$ over all straight lines $m$ is a measure of axial symmetry of $C$. We prove that $\operatorname{masf}(P)>\frac{1}{2}$ for every parallelogram $P$ and that this inequality cannot be improved.
\end{abstract}

Keywords Convex body $\cdot$ Folding $\cdot$ Measure of axial symmetry $\cdot$ Parallelogram

Mathematics Subject Classification (2000) $\quad 52 \mathrm{~A} 10 \cdot 52 \mathrm{~A} 38$

Lassak (2003) proposes the measure

$$
\operatorname{masf}(C)=2 \cdot \frac{\max _{m} \operatorname{area}\left(C_{m}\right)}{\operatorname{area}(C)}
$$

of axial symmetry of a convex body $C$ in the Euclidean plane $E^{2}$. We call it measure of axial symmetry with respect to folding. Here $C_{m}$ denotes a subset of $C$ cut off by a straight line $m$, which remains in $C$ after folding it along $m$. Moreover, in Lassak (2003) there is proved that for every convex body $C \subset E^{2}$ we have $\operatorname{masf}(C) \geq \frac{1}{4}$ (see Theorem 3 there and the Erratum). We conjecture that $\operatorname{masf}(C)>\frac{1}{2}$ for every centrally symmetric convex body $C \subset E^{2}$, and that this inequality cannot be improved in general for centrally symmetric convex bodies. We prove this inequality for parallelograms and show that it cannot be improved in general for such 4-gons.

M. Nowicka ( $\square)$

University of Technology, Kaliskiego 7, 85-789 Bydgoszcz, Poland

e-mail: mnowicka@utp.edu.pl 
A final estimate of axial symmetry of parallelograms for another kind of axial symmetry is given in Lassak and Nowicka (2010). Some other measures of axial symmetry are considered by Chakerian and Stein (1965), deValcourt (1966a,b), and Nohl (1962). A survey on measures of symmetry of convex bodies is given by Grünbaum (1963). See also Part 4.2 of the article by Heil and Martini (1993).

We omit the easy proofs of the following claims.

Claim 1 For every axially symmetric convex body $C \subset E^{2}$ the straight line $m$ for which area $\left(C_{m}\right)$ is maximal is an axis of symmetry of $C$.

Claim 2 For every convex body $C \subset E^{2}$ we have masf $(C)=1$ if and only if $C$ is axially symmetric.

We use the same symbol for an angle and its measure, and we simply say angle instead of measure of an angle. Denote by $a, b, c$ and $d$ successive vertices of the parallelogram $P$. Assume that $\angle c b a \leq \frac{\pi}{2}$ and $|a b| \leq|b c|$. By $o$ we denote the center of symmetry of $P$. Put $\alpha=\angle c b a$ and $\beta=\angle a c b$. By $\mathcal{A}$ (respectively, by $\mathcal{B}$ ) we mean the pencil of supporting lines through $a$ (respectively, through $b$ ). The symbol bi $(\angle$ efg $)$ stands for the straight line containing the bisector of the angle $L e f g$. By a strip we mean the convex hull of two parallel lines.

Let $l \in \mathcal{A} \cup \mathcal{B}$. Take an arbitrary straight line $m$ parallel to $l$ which has nonempty intersection with $P$. By $R_{m}$ we denote the subset of $P$ which is contained in the strip between $m$ and $l$. Moreover, by $T_{m}$ we mean the image of $R_{m}$ after folding it along $m$. Notice that $T_{m}$ is the mirror image of $R_{m}$ in the line $m$.

Lemma 1 Consider a parallelogram $P$ and the family of all straight lines $m$ parallel to a fixed straight line $l$ which have nonempty intersection with $P$. The union of the lines for which $T_{m} \subset P$ is a strip.

Proof The easy proof of the case when $P$ is axially symmetric is left to the reader. From now on assume that $P$ does not have an axis of symmetry. Let $l \in \mathcal{A}$ (for $l \in \mathcal{B}$, the consideration is analogous).

Let $w$ (respectively, $v$ ) be the intersection point of the segment $b c$ with the circle of radius $|a b|$ (respectively, $|a d|)$ centered at $b$ (respectively, $d$ ). Denote by $l_{1}$ and $l_{2}$ the straight lines from $\mathcal{A}$ which are parallel to bi $(\angle a d v)$ and bi $(\angle w b a)$, respectively. Since $w \in b c$, we see that $\operatorname{bi}(\angle w b a)=\operatorname{bi}(\angle c b a)$. The symbols $a_{m}, b_{m}, c_{m}$ and $d_{m}$ stand for the points correspondingly symmetric to the points $a, b, c$ and $d$ with respect to $m$. Denote by $a^{\prime}$ the intersection of the straight line through $a$ perpendicular to $l$ with the segments $b c$ or $c d$. Let $a^{\prime \prime}$ be the midpoint of $a a^{\prime}$. The points of intersection of $m$ with the boundary of $P$ are denoted by $p_{m}$ and $q_{m}$. The notation is chosen such that $p_{m} \in a d \cup c d$ and $q_{m} \in a b \cup b c$.

Case 1, when the angle between $l$ and the straight line containing the segment ad is at most the angle between $l_{1}$ and this straight line.

Denote by $k, k_{1}, k_{2}$ and $k_{3}$ the straight lines parallel to $l$ which pass through $d, a^{\prime \prime}$, $b$ and $c$, respectively. By $S, S_{1}, S_{2}$ and $S_{3}$ we mean the strips between $l$ and $k, k$ and $k_{1}, k_{1}$ and $k_{2}$ and $k_{2}$ and $k_{3}$, respectively.

Observe that for $m \in S$ we have $T_{m}=a_{m} p_{m} q_{m}$, where $p_{m} \in a d$ and $q_{m} \in a b$ (see Fig. 1). Since $p_{m}, q_{m}, a_{m}$ belong to $P$, we get $T_{m} \subset P$. 


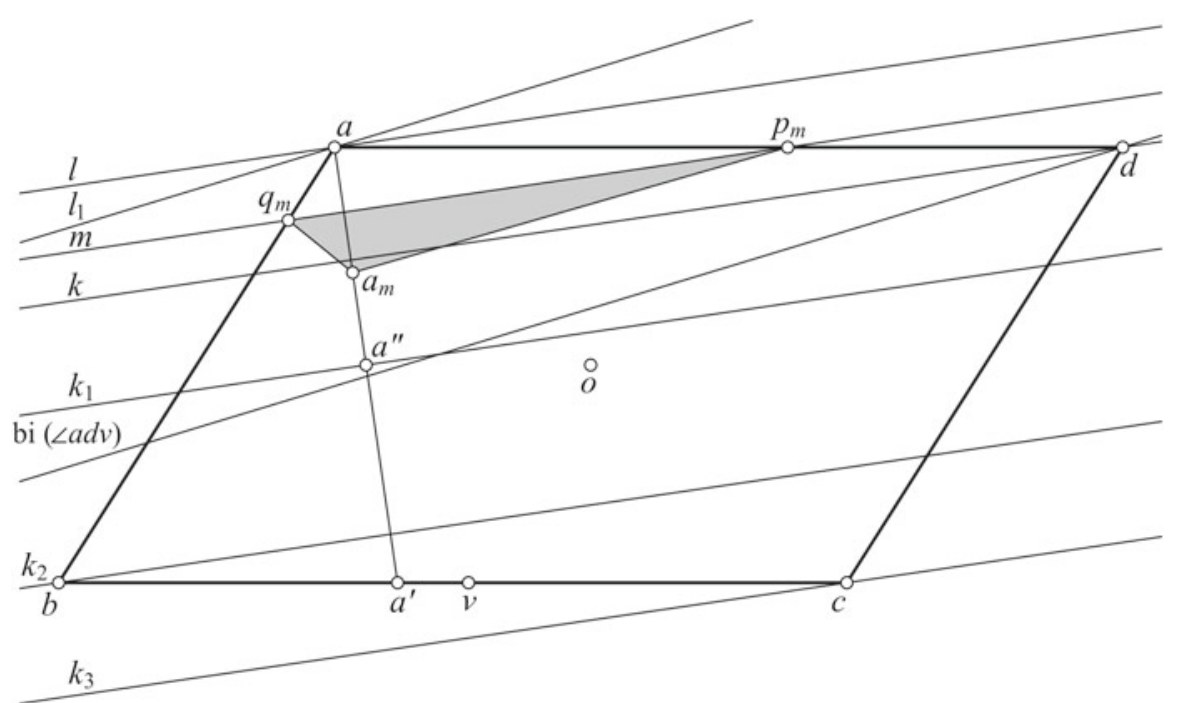

Fig. 1 The situation in Case 1 for $m \in S$

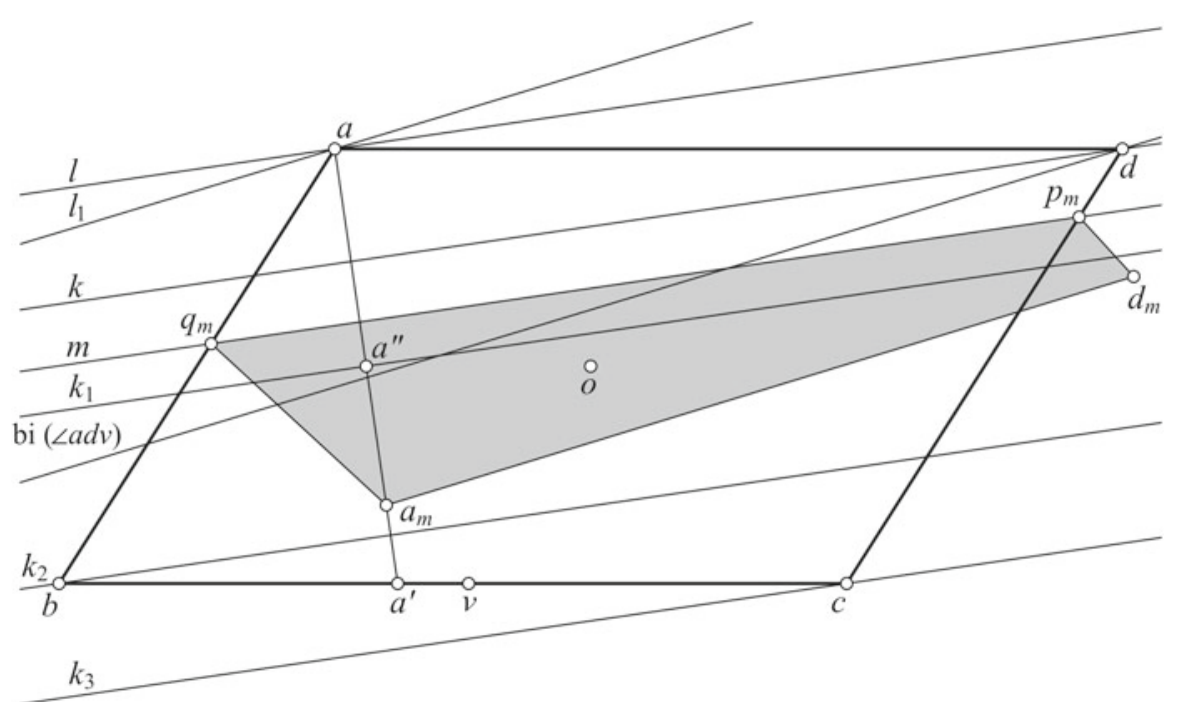

Fig. 2 The situation in Case 1 for $m \in S_{1}$

Let $m \in S_{1} \cup S_{2} \cup S_{3}$. If $m \in S_{1}$ (see Fig. 2) or $m \in S_{2}$, then $T_{m}=a_{m} d_{m} p_{m} q_{m}$, where $p_{m} \in c d$ and $q_{m} \in a b$. If $m \in S_{3}$, then $T_{m}=a_{m} d_{m} p_{m} q_{m} b_{m}$, where $p_{m} \in c d$ and $q_{m} \in b c$. For every $m \neq k$ we have $d_{m} \notin P$. Consequently, $T_{m} \not \subset P$.

Case 2, when the angle between the straight line containing ad and $l$ is greater than the angle between the straight line containing ad and $l_{1}$ and simultaneously at most the angle between the line containing ad and $l_{2}$.

Assume that the distance between $b$ and $l$ is smaller than the distance between $d$ and $l$. In the opposite situation the considerations are analogous. The symbols $k, k_{1}, k_{2}$ 
and $k_{3}$ stand for the straight lines parallel to $l$ passing through $a^{\prime \prime}, b, d$ and $c$, respectively. The strips between $l$ and $k, k$ and $k_{1}, k_{1}$ and $k_{2}, k_{2}$ and $k_{3}$ are denoted by $S, S_{1}$, $S_{2}, S_{3}$, respectively.

For $m \in S$ we have $T_{m}=a_{m} p_{m} q_{m}$. From $p_{m} \in a d, q_{m} \in a b$ and $a_{m} \in P$, we obtain $T_{m} \subset P$.

Notice that $T_{m}=a_{m} p_{m} q_{m}$ if $m \in S_{1}$, and $T_{m}=a_{m} p_{m} q_{m} b_{m}$ if $m \in S_{2}$. Moreover, if $m \in S_{3}$, then $T_{m}=a_{m} d_{m} p_{m} q_{m} b_{m}$. In the first case $p_{m} \in a d$ and $q_{m} \in a b$, in the second case $p_{m} \in a d$ and $q_{m} \in b c$, and in the third case $p_{m} \in c d$ and $q_{m} \in b c$. Observe that for every $m \neq k$ we have $a_{m} \notin P$. Hence $T_{m} \not \subset P$.

Case 3, when the angle between the straight line containing ad and $l$ is greater than the angle between the straight line containing ad and $l_{2}$.

We repeat the considerations of Case 1 taking this time instead of $k, k_{1}, k_{2}$ and $k_{3}$ the straight lines parallel to $l$ which pass through $b, a^{\prime \prime}, d$ and $c$, respectively.

From now on, for every $l \in \mathcal{A} \cup \mathcal{B}$ we denote by $S_{l}$ the strip of all straight lines $m$ parallel to $l$ for which $T_{m}$ remains in $P$.

Lemma 2 Let $P$ be a parallelogram and let $l \in \mathcal{A} \cup \mathcal{B}$. Assume that $m_{1}$ and $m_{2}$ are the straight lines parallel to $l$ such that $m_{1}, m_{2} \in S_{l}$. If the distance between $m_{1}$ and $o$ is at least the distance between $m_{2}$ and $o$, then area $\left(R_{m_{1}}\right) \leq \operatorname{area}\left(R_{m_{2}}\right)$.

Proof Without loss of generality assume that $l \in \mathcal{A}$. The proof of the case when $P$ is axially symmetric is left to the reader. We look at Cases 1-3 of Lemma 1 and we conclude that $T_{m_{1}}=a_{m_{1}} p_{m_{1}} q_{m_{1}}$ and $T_{m_{2}}=a_{m_{2}} p_{m_{2}} q_{m_{2}}$, where $p_{m_{1}}$ and $p_{m_{2}}$ are the intersections of the segment $a d$ with $m_{1}$ and $m_{2}$, respectively. Analogously, $q_{m_{1}}$ and $q_{m_{2}}$ are the intersections of the segment $a b$ with $m_{1}$ and $m_{2}$, respectively. Recall that $T_{m_{1}}$ and $T_{m_{2}}$ are the images of $R_{m_{1}}$ and $R_{m_{2}}$ after folding them along $m_{1}$ and $m_{2}$, respectively. Hence $R_{m_{1}}=a q_{m_{1}} p_{m_{1}}$ and $R_{m_{2}}=a q_{m_{2}} p_{m_{2}}$. Since $m_{1}, m_{2}$ are parallel and since the distance between $m_{1}$ and $o$ is at least the distance between $m_{2}$ and $o$, we see that $\left|a p_{m_{1}}\right| \leq\left|a p_{m_{2}}\right|$ and $\left|a q_{m_{1}}\right| \leq\left|a q_{m_{2}}\right|$. These inequalities and the fact that $\angle q_{m_{1}} a p_{m_{1}}=\angle q_{m_{2}} a p_{m_{2}}$ imply that $\operatorname{area}\left(R_{m_{1}}\right) \leq \operatorname{area}\left(R_{m_{2}}\right)$.

From Lemmas 1 and 2 we get the following corollary.

Corollary 1 For a given strip $S_{l}$ the maximum area of $R_{m}$ is attained for that straight line $m$ bounding $S_{l}$ which is closer to o.

Below we find the straight lines cutting off from $P$ the subsets of the largest areas which remain in $P$ after folding them along those straight lines. Observe that, if $P$ is axially symmetric, then by Claim 1 they are the axes of symmetry of $P$. Hence in Lemma 3 we assume that $P$ is not an axially symmetric parallelogram. Without loss of generality, we assume that in $P$ we have $|b c|=1$. For further considerations we need the Cartesian coordinate system $O x y$ in which $a=(p, q), b=(0,0), c=(1,0)$ and $d=(p+1, q)$, where $p \in(0,1)$ and $q \in\left(0, \sqrt{1-p^{2}}\right)$.

Lemma 3 Let $P$ be a parallelogram which is not axially symmetric and whose longer side has length 1 . Then $\max _{m}\left\{\operatorname{area}\left(R_{m}\right) ; T_{m} \subset P\right\}=\max \left\{\operatorname{area}\left(R_{\mathrm{bi}(L a d v)}\right)\right.$, $\left.\operatorname{area}\left(R_{\mathrm{bi}(L w b a)}\right)\right\}=\max \left\{\frac{1}{2\left(1-p+\sqrt{1-q^{2}}\right)}, \frac{\sqrt{p^{2}+q^{2}}}{2}\right\} \cdot \operatorname{area}(P)$. 
Proof Let $l \in \mathcal{A} \cup \mathcal{B}$. Denote by $\varphi$ the angle between the straight line $l$ and the axis $O x$. Having in mind the Corollary, we limit the consideration to a straight line $m \in S_{l}$ bounding $S_{l}$ which is closer to $o$. Of course, the angle between $m$ and $O x$ is also $\varphi$.

Case 1, when $\varphi \in[0, \alpha]$.

Subcase 1.1, when $\varphi \leq \frac{1}{2} \angle a d v$.

Clearly, $S_{l}$ is the strip between $l$ and the parallel straight line $m$ passing through $d$. Hence $R_{m}=a q_{m} d$, where $q_{m} \in a b$. Observe that if $\varphi$ increases from 0 to $\frac{1}{2} \angle a d v$, then $\left|d q_{m}\right|$ and $L a d q_{m}=\varphi$ are increasing and $|a d|$ is constant. Consequently, area $\left(R_{m}\right)$ increases and attains its greatest value for $m=\operatorname{bi}(\angle a d v)$.

Subcase 1.2, when $\frac{1}{2} L a d v \leq \varphi \leq \frac{1}{2} \angle w b a$.

Since $\frac{1}{2} \angle a d v>0$ and $\frac{1}{2} \angle w b a=\frac{\alpha}{2}$, we conclude that $\varphi \in\left(0, \frac{\alpha}{2}\right]$. In this subcase $S_{l}$ is the strip between $l$ and the parallel straight line $m$ which passes through the midpoint $a^{\prime \prime}$ of $a a^{\prime}$. Observe that $R_{m}=a q_{m} p_{m}$ and $T_{m}=a_{m} p_{m} q_{m}$, where $p_{m}$ and $q_{m}$ are the points of intersection of $m$ with $a d$ and $a b$, respectively. Assume that the equation of $m$ is $y=A x+B$. From $a_{m} \in b c$ we obtain $a_{m}=(A q+p, 0)$. Of course, $B=\frac{1}{2}\left(q-2 A p-A^{2} q\right)$. Having in mind that $p_{m} \in a d$ and providing easy calculation, we obtain that $p_{m}=\left(\frac{q-B}{A}, q\right)$. Since $q_{m} \in a b$, we have $q_{m}=\left(\frac{B}{q / p-A}, q / p \frac{B}{q / p-A}\right)$. We see that the area of $R_{m}$ equals

$$
f(A)=\frac{q^{3}}{8 p} \frac{\left(A^{2}+1\right)^{2}}{(q / p-A) A} .
$$

Clearly, $A=\frac{\sin \varphi}{\cos \varphi}$. Moreover, it is easy to show that $p=\frac{\cos \alpha \sin \beta}{\sin (\alpha+\beta)}$ and $q=\frac{\sin \alpha \sin \beta}{\sin (\alpha+\beta)}$. Substituting these three equalities into (1) and providing elementary, but time-consuming calculation, we obtain

$$
f(\varphi)=\frac{\sin ^{3} \alpha \sin ^{2} \beta}{\sin ^{2}(\alpha+\beta)} \cdot \frac{1}{2 \sin \alpha \sin 2 \varphi+\cos (\alpha-4 \varphi)-\cos \alpha} .
$$

Consider the function $g(\varphi)=2 \sin \alpha \sin 2 \varphi+\cos (\alpha-4 \varphi)-\cos \alpha$. Since $g^{\prime \prime}(\varphi)=$ $-8 \sin \alpha \sin 2 \varphi-16 \cos (\alpha-4 \varphi)$ is negative in the interval $\left(0, \frac{\alpha}{2}\right]$, we see that $g(\varphi)$ is concave in this interval. Hence the function $f(\varphi)$ is convex in the interval $\left(0, \frac{\alpha}{2}\right]$. This and $\left[\frac{1}{2} L a d v, \frac{1}{2} L w b a\right] \subset\left(0, \frac{\alpha}{2}\right]$ imply that $f(\varphi)$ attains the greatest value at one or both endpoints of the interval $\left[\frac{1}{2} \angle a d v, \frac{1}{2} \angle w b a\right]$. So the area of $R_{m}$ may be maximal only for $m=\operatorname{bi}(\angle a d v)$, or only for $m=\operatorname{bi}(\angle w b a)$, or for both $m=\operatorname{bi}(\angle a d v)$ and $m=\operatorname{bi}(\angle w b a)$.

Subcase 1.3, when $\varphi \geq \frac{1}{2} \angle w b a$.

Now $S_{l}$ is the strip between $l$ and the parallel straight line $m$ passing through $b$. Of course, $R_{m}=a b p_{m}$, where $p_{m} \in a d$. When $\varphi$ increases from $\frac{1}{2} \angle w b a$ to $\angle c b a$, then $\left|b p_{m}\right|$ and $\angle p_{m} b a=\alpha-\varphi$ decrease and $|a b|$ is constant. We conclude that area $\left(R_{m}\right)$ decreases.

Case 2, when $\varphi \in[\alpha, \pi]$.

Similarly like in Case 1 we show that the area of $R_{m}$ attains its maximum for $m=$ $\operatorname{bi}(\angle b a d)$. 
From Cases 1 and 2 and next by $\operatorname{area}\left(R_{\mathrm{bi}\left(L_{w b a}\right)}\right)=\operatorname{area}\left(R_{\mathrm{bi}(\angle b a d)}\right)$ we obtain that

$$
\begin{aligned}
\max _{m} \operatorname{area}\left(R_{m}\right) & =\max \left\{\operatorname{area}\left(R_{\mathrm{bi}(\angle a d v)}\right), \operatorname{area}\left(R_{\mathrm{bi}(\angle w b a)}\right), \operatorname{area}\left(R_{\mathrm{bi}(\angle b a d)}\right)\right\} \\
& =\max \left\{\operatorname{area}\left(R_{\mathrm{bi}(\angle a d v)}\right), \operatorname{area}\left(R_{\mathrm{bi}(\angle w b a)}\right)\right\}
\end{aligned}
$$

Below we calculate area $\left(R_{\mathrm{bi}(L a d v)}\right)$. Having in mind that $v$ is the intersection point of the segment $b c$ with the circle of radius $|a d|=1$ centered at $d$, straightforward calculation shows that $v=\left(p+1-\sqrt{1-q^{2}}, 0\right)$. Since bi $(\angle a d v)$ contains the bisector of the angle $L a d v$ we conclude that its slope equals $\frac{1-\sqrt{1-q^{2}}}{q}$. Taking $A=\frac{1-\sqrt{1-q^{2}}}{q}$ in (1) we have $f\left(\frac{1-\sqrt{1-q^{2}}}{q}\right)=\frac{q}{2\left(1-p+\sqrt{1-q^{2}}\right)}$. Consequently, area $\left(R_{\mathrm{bi}(L a d v)}\right)=$ $\frac{q}{2\left(1-p+\sqrt{1-q^{2}}\right)}$.

Similar considerations like above show that $\operatorname{area}\left(R_{\mathrm{bi}(\angle w b a)}\right)=\frac{q \sqrt{p^{2}+q^{2}}}{2}$.

From the preceding three paragraphs and from area $(P)=q$ we get max area $\left(R_{m}\right)=\max \left\{\operatorname{area}\left(R_{\mathrm{bi}(\angle a d v)}\right), \operatorname{area}\left(R_{\mathrm{bi}(\angle w b a)}\right)\right\}=\max \left\{\frac{1}{2\left(1-p+\sqrt{1-q^{2}}\right)}, \frac{\sqrt{p^{2}+q^{2}}}{2}\right\}$. $\operatorname{area}(P)$.

By the definition of $\operatorname{masf}(C)$ and from Lemma 3 we obtain the following proposition.

Proposition For every parallelogram $P$ which is not axially symmetric and whose longer side has length 1 we have $\operatorname{mas} f(P)=\max \left\{\frac{1}{1-p+\sqrt{1-q^{2}}}, \sqrt{p^{2}+q^{2}}\right\}$, where $p$ and $q$ are defined just like before Lemma 3.

Theorem For every parallelogram $P$ we have masf $(P)>\frac{1}{2}$. In general, this inequality cannot be improved.

Proof Since the measure of axial symmetry is invariant under homotheties, below we consider only parallelograms defined as before Lemma 3. Of course, our family does not contain axially symmetric parallelograms (from Claim 2 it follows that $\operatorname{masf}(P)=$ 1 for each of them, and thus Theorem holds true for them). It is easy to show that $\frac{1}{1-p+\sqrt{1-q^{2}}}$ and $\sqrt{p^{2}+q^{2}}$ decrease provided $q \in\left(0, \sqrt{1-p^{2}}\right)$ decreases and $p$ is fixed. Observe that $\lim _{q \rightarrow 0} \frac{1}{1-p+\sqrt{1-q^{2}}}=\frac{1}{2-p}$ and $\lim _{q \rightarrow 0} \sqrt{p^{2}+q^{2}}=p$. Moreover for every $p \in(0,1)$ we have $\max \left\{\frac{1}{2-p}, p\right\} \geq \frac{1}{2-p}>\frac{1}{2}$. In other words, $\lim \operatorname{masf}(P)=\frac{1}{2}$ provided $p \rightarrow 0$ and $q \rightarrow 0$.

Consider the family of parallelograms for which $p=\mu, q=\sqrt{2 \mu-\mu^{2}}$ and $\mu \in\left(0, \frac{3-\sqrt{5}}{4}\right)$. Then $\operatorname{masf}(P)=\max \left\{\frac{1}{2-2 \mu}, \sqrt{2 \mu}\right\}$. For every $\mu$ from $\left(0, \frac{3-\sqrt{5}}{4}\right)$ we have $\frac{1}{2-2 \mu}>\sqrt{2 \mu}$. So $\operatorname{masf}(P)=\frac{1}{2-2 \mu}$. If $\mu \rightarrow 0$, then $\operatorname{masf}(P) \rightarrow \frac{1}{2}$. Hence this family of parallelograms shows that, in general, the inequality presented in the Theorem cannot be improved. 
Remark In order to show the second part of the Theorem we may also take some other families of parallelograms for which $p$ and $q$ tend to 0 , e.g., the family for which $p=q$.

Open Access This article is distributed under the terms of the Creative Commons Attribution Noncommercial License which permits any noncommercial use, distribution, and reproduction in any medium, provided the original author(s) and source are credited.

\section{References}

Chakerian, G.D., Stein, S.K.: Measures of symmetry of convex bodies. Canad. J. Math. 17, 497-504 (1965). Zbl 0137.15302

deValcourt, B.A.: Measures of axial symmetry for ovals. Bull. Amer. Math. Soc. 72, 289-290 (1966a). Zbl 0149.19301

deValcourt, B.A.: Measures of axial symmetry for ovals. Israel J. Math. 4, 65-82 (1966b). Zbl 0144.44201

Grünbaum, B.: Measures of symmetry of convex bodies. Proc. Sympos. Pure Math. 7, 233-270 (1963). Zbl 0142.20503

Heil, E., Martini, H.: Special Convex Bodies. In: P.M. Gruber, J.M. Wills (eds) Handbook of Convex Geometry, vol. A, pp. 347-385. North-Holland, Amsterdam 1993. Zbl 0794.52002

Lassak, M.: Approximation of convex bodies by axially symmetric bodies. Proc. Amer. Math. Soc. 130, 3075-3084 (2002). Erratum in 131, pp. 2301 (2003). Zbl 1007.52001

Lassak, M., Nowicka, M.: A measure of axial symmetry of centrally symmetric convex bodies. Colloq. Math. 121, 295-306 (2010)

Nohl, W.: Die innere axiale Symmetrie zentrischer Eibereiche der Euklidischen Ebene. Elem. Math. 17 , 59-63 (1962). Zbl 0118.17304 